\title{
Performance Evaluation of Coherence-Based Adaptive Imaging Using Clinical Breast Data
}

\author{
Shun-Li Wang, Chen-Han Chang, Hsin-Chia Yang, Yi-Hong Chou, and Pai-Chi Li, Senior Member, IEEE
}

\begin{abstract}
Sound-velocity inhomogeneities degrade both the spatial resolution and the contrast in diagnostic ultrasound. We previously proposed an adaptive imaging approach based on the coherence of the data received in the channels of a transducer array, and we tested it on phantom data. In this study, the approach was tested on clinical breast data and compared with a correlation-based method that has been widely reported in the literature. The main limitations of the correlation-based method in ultrasonic breast imaging are the use of a near-field, phasescreen model and the integration errors due to the lack of a two-dimensional (2-D) array. In contrast, the proposed coherence-based method adaptively weights each image pixel based on the coherence of the receive-channel data. It does not make any assumption about the source of the focusing errors and has been shown to be effective using 1-D arrays. This study tested its in vivo performance using clinical breast data acquired by a programmable system with a $5 \mathrm{MHz}$, 128-channel linear array. Twenty-five cases (6 fibroadenomas, 10 carcinomas, 6 cysts, and 3 abscesses) were investigated. Relative to nonweighted imaging, the average improvements in the contrast ratio and contrast-tonoise ratio for the coherence-based method were $8.57 \mathrm{~dB}$ and $23.2 \%$, respectively. The corresponding improvements when using the correlation-based method were only $0.42 \mathrm{~dB}$ and $3.35 \%$. In an investigated milk-of-calcium case, the improvement in the contrast was $4.47 \mathrm{~dB}$ and the axial and lateral dimensions of the object were reduced from 0.39 to $0.32 \mathrm{~mm}$ and from 0.51 to $0.43 \mathrm{~mm}$, respectively. These results demonstrate the efficacy of the coherencebased method for clinical ultrasonic breast imaging using 1-D arrays.
\end{abstract}

\section{INTRODUCTION}

$\mathrm{A}$ PRIMARY performance limitation of diagnostic ultrasound is the distortion introduced by sound-velocity inhomogeneities in human tissue. Because the sound velocity varies over a wide range (e.g., $1450 \mathrm{~m} / \mathrm{s}$ in fat and $1665 \mathrm{~m} / \mathrm{s}$ in collagen [1]), the use of a constant sound velocity when calculating focusing delays inevitably results in focusing errors. Such focusing errors also are termed phase aberrations, and they degrade both the spatial resolution and the contrast in the resultant images.

Manuscript received October 4, 2006; accepted March 2, 2007. Support from the National Science Council of Taiwan under grant no. NSC 95-2221-E-002-170 is gratefully acknowledged.

S.-L. Wang, C.-H. Chang, H.-C. Yang, and P.-C. Li are with the Department of Electrical Engineering, National Taiwan University, Taipei, Taiwan.

Y.-H. Chou is with the Department of Radiology, Taipei Veterans General Hospital, Taipei, Taiwan.

P.-C. Li also is with the Graduate Institute of Biomedical Electronics and Bioinformatics, National Taiwan University, Taipei, Taiwan (e-mail: paichi@cc.ee.ntu.edu.tw).

Digital Object Identifier 10.1109/TUFFC.2007.438
Several phase-aberration correction techniques have been proposed to adaptively reduce these errors and thereby improve the image quality [2]-[15]. Flax and O'Donnell [2] proposed a correlation-based method that modeled sound-velocity inhomogeneities as a near-field phase screen, which assumes that time-delay errors produce phase aberrations only at the surface of the transducer. The near-field phase screen can be estimated by finding the peak of the cross-correlation function of the signals from adjacent channels [2], [3], or using those from the individual channels to the beam sum [4] or partial beam sum [5]. Phase aberrations theoretically can be corrected by compensating for the time-delay errors, but several factors have limited the clinical applications of correlationbased methods. First, the near-field phase-screen model is often inadequate because sound-velocity inhomogeneities result in not only time-delay errors but also energy-level fluctuations and waveform distortion. This led to a displaced phase-screen model that accounts for phase aberrations as a phase screen positioned some distance from the transducer surface [6]. However, its performance in ultrasonic breast imaging is still insufficient because the soundvelocity variations in breast are more distributed. The other reason for the inadequate performance of correlationbased methods is the three-dimensional (3-D) nature of sound-velocity inhomogeneities. Preventing aberrator integration errors in time-delay estimation requires the use of a 1.5-D or 2-D array [7], [16]. However, these are not yet in widespread use in clinics due to the significantly increased system complexity and cost.

Adaptive sidelobe-reduction techniques also can be used to reduce focusing imperfections [8]-[15], one example of which is the parallel adaptive receive-compensation algorithm (PARCA) [8]-[11]. At each image point, the PARCA removes unwanted sidelobe contributions and maintains the mainlobe signal intensity by estimating the on-axis and off-axis signal components. However, a significant drawback of PARCA is its computational complexity.

Another adaptive sidelobe-reduction method is based on the use of the coherence factor $(\mathrm{CF})$ of the receivechannel data [12]-[14], which represents a focusing-quality index:

$$
\mathrm{CF}=\frac{\left|\sum_{i=0}^{N-1} S(i, t)\right|^{2}}{N \sum_{i=0}^{N-1}|S(i, t)|^{2}} .
$$


where $N$ denotes the total number of array channels, $S(i)$ is the signal received at channel $i$, and $t$ denotes the time corresponding to the depth of the image point. In other words, the $\mathrm{CF}$ is defined as the ratio between the energy of the coherent sum to the total incoherent energy (i.e., $N$ times the incoherent sum). Therefore, an image point with a high $\mathrm{CF}$ value possesses good focusing quality, and its amplitude should be maintained, whereas the amplitude of an image point with a low $\mathrm{CF}$ value possesses poor focusing quality and its amplitude should be suppressed. Thus, this method is effectively an adaptive weighting technique in which the amplitude of each image pixel is weighted by the corresponding $\mathrm{CF}$ such that the unwanted sidelobes are effectively reduced. The $\mathrm{CF}$ also has been modified to the generalized $\mathrm{CF}$ (GCF) to accommodate the coherence properties of speckle-generating objects [15]. The efficacy of the GCF-weighting method has been demonstrated for phantom data [15]. The main purpose of the present study was to test the feasibility of applying the GCF to clinical breast data. Specifically, the method was applied to different types of breast lesions: cyst, fibroadenoma, carcinoma, and abscess.

This paper explores the contrast and other general characteristics of the GCF technique. It is organized as follows. In Section II, the basic principles and experimental methods for the GCF-weighting method are described. Section III presents the experimental results. The results then are discussed in Section IV. The paper concludes in Section V.

\section{Imaging Principles and Research Methods}

\section{A. Generalized Coherence Factor}

The GCF is calculated using the Fourier spectrum of the aperture-domain data, which are the data received in individual array channels after the application of focusing delays but prior to beam summation. The corresponding Fourier spectrum is obtained by performing a discrete Fourier transform across the array at each time (i.e., depth):

$$
S(i, t) \stackrel{\mathrm{FT}}{\longrightarrow} p(k, t)
$$

where $p(k, t)$ is the Fourier spectrum with spatial frequency index $k$ and time variable $t$. As described in [11], the spectrum can be viewed as an approximation of multiple parallel receive beams centered at the transmit beam direction. The definition of the $\mathrm{CF}$ described in (1) also can be expressed as follows:

$$
\mathrm{CF}=\frac{|p(0, t)|^{2}}{\sum_{k=-N / 2}^{N / 2-1}|p(k, t)|^{2}}
$$

The numerator of (3) is the energy of the dc component of the spectrum, and the denominator is the total spectral energy. The dc component represents the signals received from the transmit beam direction (i.e., on-axis component). The other components represent the signals from other angles (i.e., off-axis components).

Adaptive weighting using $\mathrm{CF}$ is feasible for pointlike targets. However, for diffuse scatterers (i.e., specklegenerating objects), part of the mainlobe signal appears within the low-frequency region. Therefore, using the ratio of the dc energy to the total energy as a weighting factor underestimates the coherence. The GCF was proposed to take this into account [15], and it thus is defined as the ratio of the energy within a certain low-frequency region to the total energy:

$$
\mathrm{GCF}=\frac{\sum_{k=-M_{0}}^{M_{0}}|p(k, t)|^{2}}{\sum_{k=-N / 2}^{N / 2-1}|p(k, t)|^{2}},
$$

where $M_{0}$ denotes the prespecified cut-off frequency in the spatial-frequency domain. Note that the CF is the special case of the GCF with $M_{0}=0$.

\section{B. Signal Processing Procedures}

The signal processing procedures we used are shown schematically in Fig. 1. After the echo signal is received and digitized by the $\mathrm{A} / \mathrm{D}$ converter, the received $\mathrm{RF}$ data are demodulated down to baseband, and the baseband beamformer applies geometric focusing delays and phase rotations to the baseband data. The B-mode image then is obtained by performing beam summation across the array with prespecified apodization. Note that the geometric delays are adjusted for every depth on receive to achieve dynamic focusing. However, as previously mentioned, the focusing delays applied in a beamformer are derived by assuming a constant sound velocity without considering the sound velocity variations in soft tissues. Therefore, it is the purpose of the proposed GCF method to estimate and suppress such focusing errors. Because the carrier frequency needs to be removed when calculating the coherence factor, baseband beamformers are preferred. The GCF is calculated for the specific configuration using the delayed baseband data across the aperture at all ranges prior to beam summation. The amplitude of the beam sum then simply is multiplied by the corresponding GCF:

$$
x_{\text {weighted }}=\mathrm{GCF} \cdot x,
$$

where $x$ denotes the original amplitude of the beam-sum data, and $x_{\text {weighted }}$ denotes the amplitude of the beam sum weighted by the GCF. The weighted beam-sum data then are stored in a buffer for further signal processing, scan conversion, and display.

\section{Clinical Data Acquisition}

Experiments were performed to evaluate the efficacy of the proposed method in clinical breast imaging. Clinical 


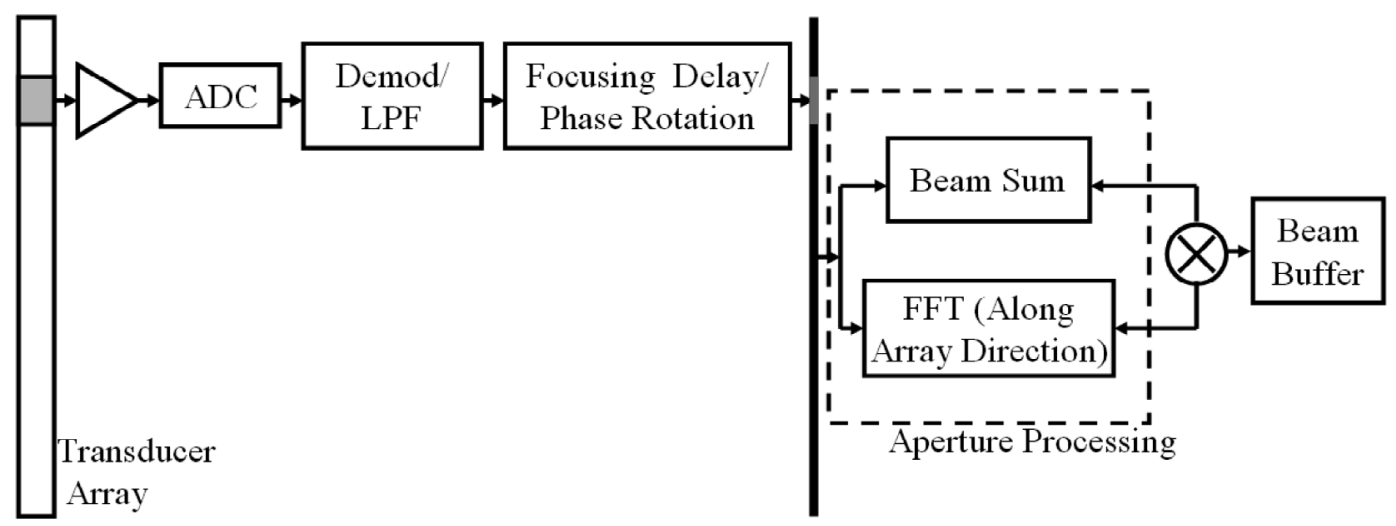

Fig. 1. Signal processing procedures of the proposed GCF-weighting technique.

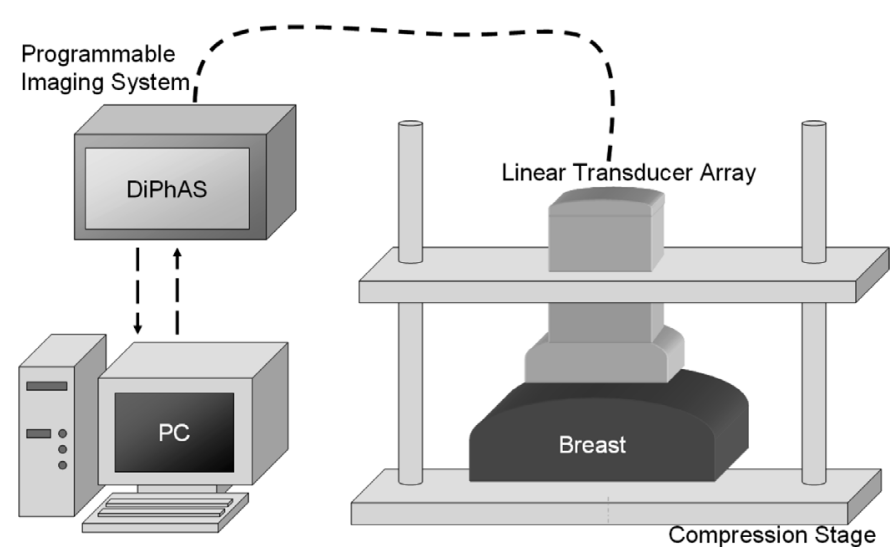

Fig. 2. Clinical data-acquisition apparatus.

data of the female breast were collected in the ultrasonic consulting room of the Taipei Veterans General Hospital by one of the authors (Y.-H. Chou) after patients had provided informed consent. A total of 25 patients aged from 23 to 89 years were included. Among them, there were 6 fibroadenomas, 10 carcinomas, 6 cysts, and 3 abscesses. A schematic of the experimental apparatus is shown in Fig. 2, which included a mechanical compression stage similar to that used in X-ray mammography to stabilize the breast during data acquisition. A programmable imaging system with 64 system channels (DiPhAS, IBMT, Fraunhofer Institutes, St. Ingbert, Germany) was used to acquire the aperture-domain data (i.e., the receive-channel data prior to beam summation). The linear transducer array had a center frequency of $5 \mathrm{MHz}, \mathrm{a}-6 \mathrm{~dB}$ bandwidth of $4.1 \mathrm{MHz}$ and 128 channels (L6/128, Sound Technology, State College, PA) at a pitch of $0.3 \mathrm{~mm}$. A complete data set was obtained from each patient (i.e., $128 \times 128 \mathrm{RF}$ Amode scans recorded from the 128-element array, for which each A-mode scan represents the echo signal of a particular transmit/receive combination) at a sampling rate of $20 \mathrm{MHz}$. Each complete data set took approximately $3 \mathrm{~s}$ to acquire. The acquired data sets were used to reconstruct the aperture-domain data with full dynamic focus on both transmit and receive. In order to evaluate tissue motion, B-mode images were acquired and stored before and after full data set acquisition. The two images then were compared to make sure that no noticeable tissue motion was present. An f-number of 1 was applied for aperture control. All images displayed here are in linear gray scale mapping with a dynamic range of $60 \mathrm{~dB}$ and are shown in a linear scan format. The step size for the linear scan was $0.15 \mathrm{~mm}$ (i.e., half the pitch of the transducers). B-mode images are formed by applying transmit and receive focusing delays before beam summation, and multiple imaging methods can be compared with one another using the same data set.

\section{Experimental Results}

\section{A. Cut-off Frequency $M_{0}$}

In the GCF technique, cut-off frequency $M_{0}$ should be specified so as to achieve optimal performance. Both the contrast ratio $(\mathrm{CR})$ and contrast-to-noise ratio (CNR) were used in the experiments to evaluate the effects of varying $M_{0}$. The $\mathrm{CR}$ is defined as the ratio between the mean value in a background region to that in a region of interest (ROI) [3]. The CNR is defined as the ratio between the CR and the standard deviation of the image intensity in a background region [4]. Fig. 3(a) shows an original image without application of the GCF-weighting method, and Figs. 3(b)-(f) show the GCF-weighted images with $M_{0}$ ranging from 0 to 4 . Note that $M_{0}=0$ represents the case of using the $\mathrm{CF}$. The background region and ROI used to calculate CRs and CNRs are indicated in Fig. 3(a) by the black and white boxes, respectively. The ROI in this case is a cyst. Note that in all cases the contrast is clearly improved, and the background noise is suppressed. Figs. 4(a) and (b) show the CR and CNR after GCF weighting as functions of $M_{0}$, respectively. Fig. 4(a) shows that the CR was improved in all cases, decreasing as $M_{0}$ increased. When the background-intensity variations are taken into account, Fig. 4(b) shows that the CNR was not improved when $M_{0}=0$. Nevertheless, with $M_{0} \geq 1$, CNR improvement is again demonstrated. Specifically, the optimal CNR was achieved at $M_{0}=3$ in this case. The results indicate that using $\mathrm{CF}$ as a weighting index can 

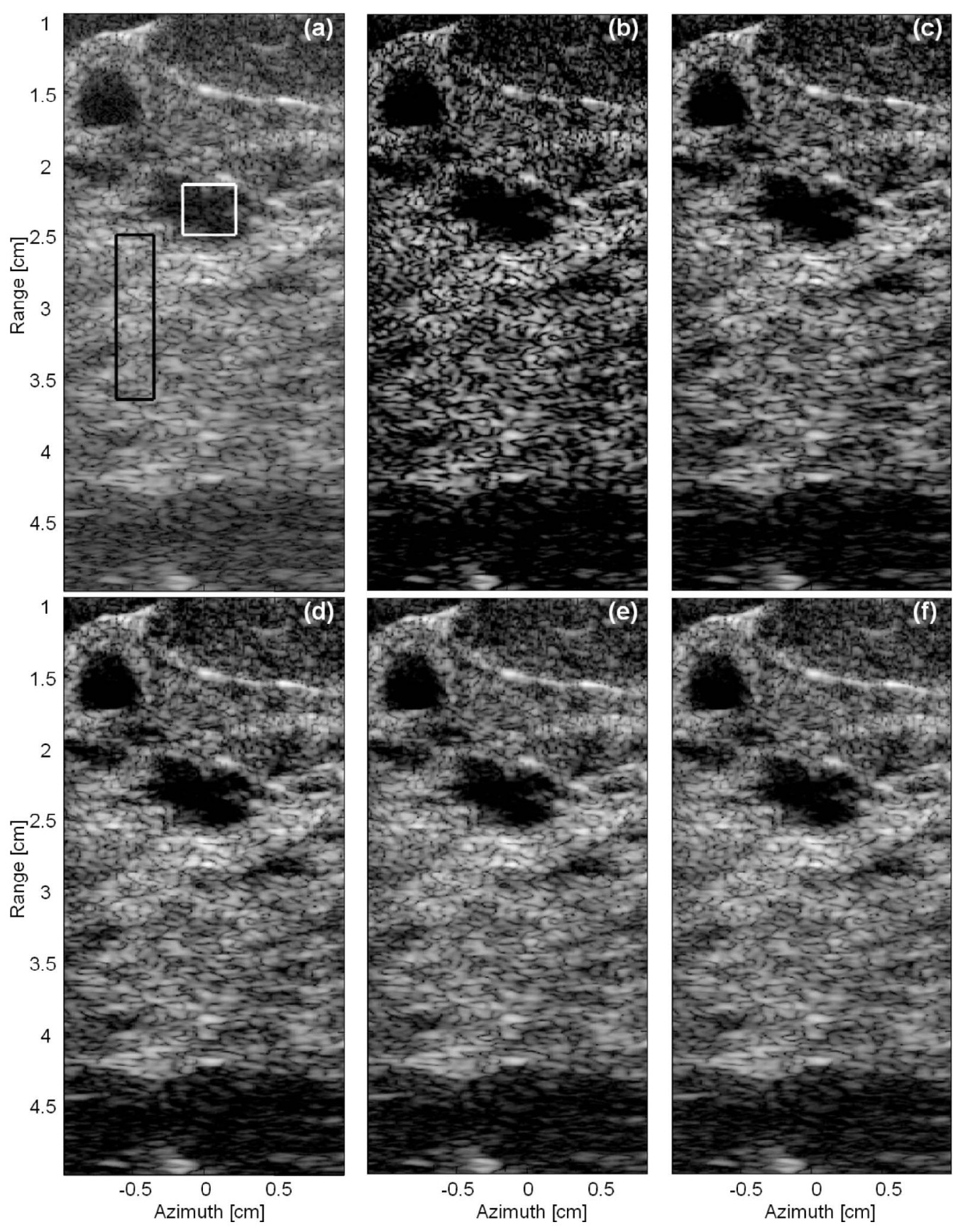

Fig. 3. Effects of cut-off frequency $M_{0}$ on the images of a a cyst, displayed with a dynamic range of 60 dB. (a) Original image. Black box indicates background region. White box indicates ROI. (b)-(f) GCF-corrected images for $M_{0}$ values of 0 (b), 1 (c), 2 (d), 3 (e), and 4 (f).

effectively increase the CR. However, CF also gives rise to strong intensity variations and degrades the CNR. This also is illustrated in Fig. 3(b) in which the CF-weighting technique introduced artifactual black holes in the background region, which in turn affect the detection of an ROI. Based on these results, $M_{0}=3$ was used in the subsequent investigations (which are described below).

\section{B. Cyst}

The sonogram needs to be interpreted accurately in order to avoid unnecessary biopsies and to differentiate a cyst from a solid mass. Fig. 5 shows images of a cyst in a 66-year-old female patient. The background and the ROI regions used in the $\mathrm{CR}$ and CNR calculations are indicated in Fig. 5(a) by the black and white boxes, respectively. Fig. 5(a) shows the original image, Fig. 5(b) shows the image obtained using the correlation-based method (as a reference) [2], [3], and Fig. 5(c) is the image obtained with adaptive compensation using GCF weighting. For the correlation-based method, time delay between adjacent channels was calculated by finding the peak position of the cross-correlation function of the signals received by the two channels. An infinite isoplanatic patch size (i.e., single aberration profile applied throughout the image) was used in this study without smoothing the aber- 


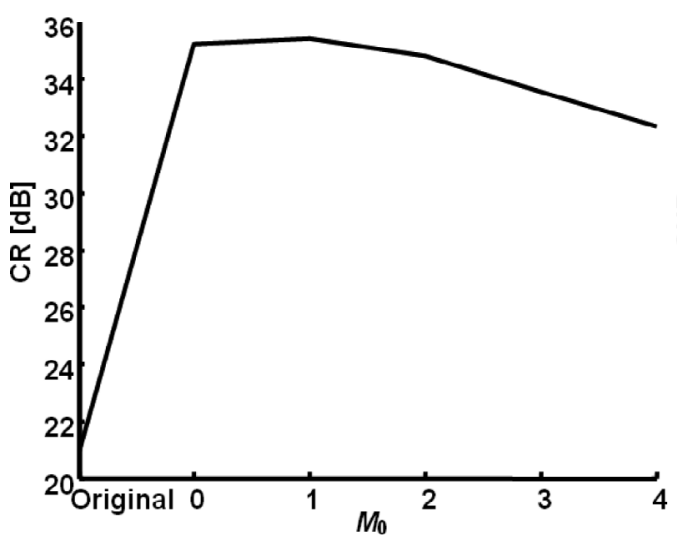

(a)

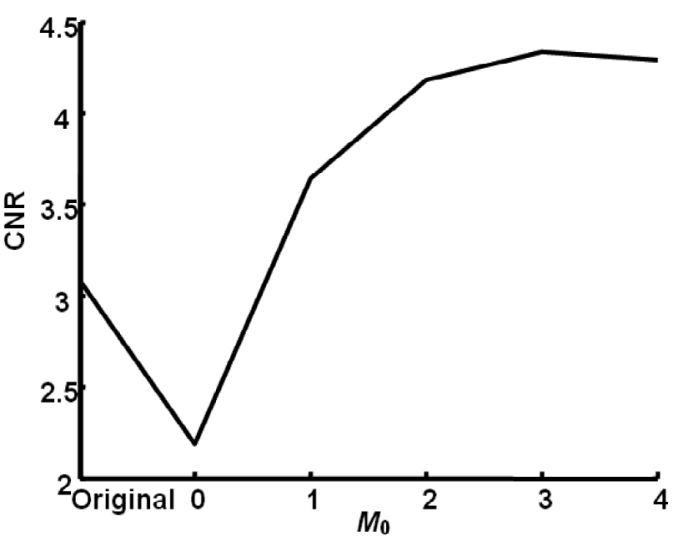

(b)

Fig. 4. Values of CR (a) and CNR (b) for the specimen shown in Fig. 3 as functions of $M_{0}$.
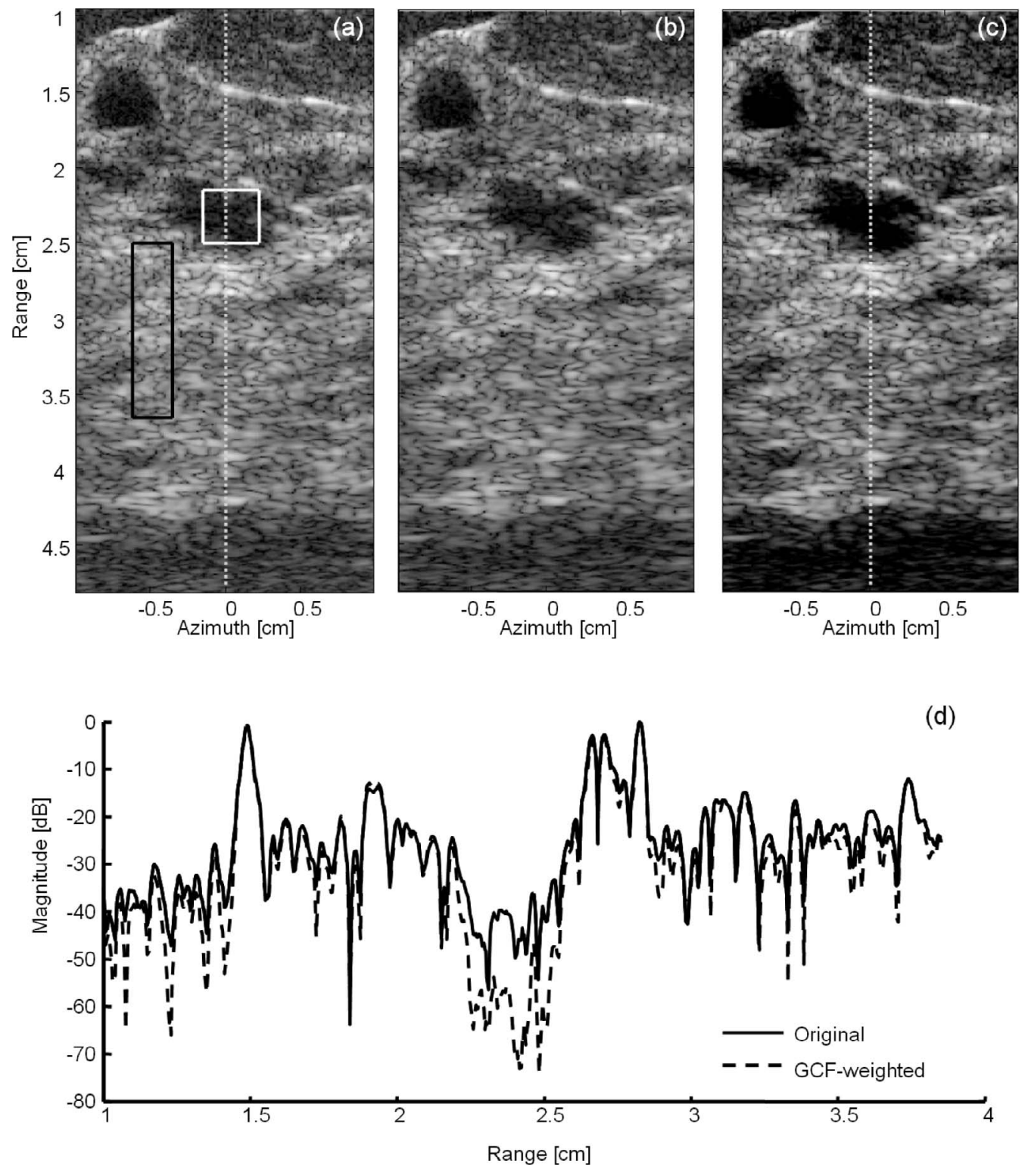

Fig. 5. Images of a cyst in a 66-year-old woman, displayed with a dynamic range of $60 \mathrm{~dB}$ : original B-mode image (a), and images obtained using the correlation-based method (b) and the GCF-weighting method (c). The black box in (a) indicates the background image. The white box indicates the ROI. (d) Intensities of the scan-line data along the gray dotted lines shown in (a) and (c) using solid and dashed lines, respectively. 
ration profile. The aberration profile is applied after the linear components were removed. The CRs and CNRs were $20.97 \mathrm{~dB}$ and 3.07 for the original image, $20.52 \mathrm{~dB}$ and 2.95 for the correlation-based method, and $33.53 \mathrm{~dB}$ and 4.33 for the GCF-weighting method. With the correlationbased method, the CR and CNR were slightly degraded, by $0.44 \mathrm{~dB}$ and $3.85 \%$, respectively. This may be due to the phase aberrations in the breast being more distributed, and aberrator integration errors may be present with the 1-D array. In contrast, the improvements in the $\mathrm{CR}$ and CNR using the GCF-weighting method were $12.56 \mathrm{~dB}$ and $40.97 \%$, respectively. The original image shows an indistinct internal echo within the cystic mass. The GCFweighted image shows that the signal within the cystic mass was effectively suppressed and that the CR was enhanced. The solid line in Fig. 5(d) shows the intensity along the scan line shown as the dotted vertical line in Fig. 5(a). With the proposed method, the intensity along this line [i.e., the vertical dotted line in Fig. 5(c)] is shown as the dashed line in Fig. 5(d). Note that image intensities between $1-1.4 \mathrm{~cm}$ and $2.2-2.5 \mathrm{~cm}$ are similar in the original image [Fig. 5(a)]. With the application of the proposed method; however, image intensities resulting from unwanted sidelobes in the cyst region $(2.2-2.5 \mathrm{~cm})$ are effectively reduced. The image intensities in the tissue region (1-1.4 cm) are less affected. Therefore, effective sidelobe reduction is demonstrated, and it is clear that the method is not just a simple "reject" control. The margin of the cyst was more distinct than that in the original image. These results illustrate the significant improvement in contrast and boundary definition in this case.

\section{Fibroadenoma}

Fig. 6 shows images of a fibroadenoma lesion in a 42year-old female patient. The background and the ROI regions used in the calculation of the CR and CNR are indicated in Fig. 6(a) by the black and white boxes, respectively. Fig. 6(a) shows the original image, and Figs. 6(b) and (c) show the corrected images obtained using the correlation-based method and the GCF-weighting method, respectively. The CRs and CNRs were $16.92 \mathrm{~dB}$ and 2.76 for the original image, $17.51 \mathrm{~dB}$ and 2.82 for the correlation-based method, and $25.73 \mathrm{~dB}$ and 3.67 for the GCF-weighting method. The CR and CNR improved by $0.58 \mathrm{~dB}$ and $2.36 \%$ with the correlation-based method and by $8.81 \mathrm{~dB}$ and $33.07 \%$ with the GCF-weighting method. The original image shows poor boundary definition. However, with the application of the correlation-based method, no noticeable change was observed, and the method was not able to improve the image. But, the use of the GCFweighting method noticeably improves the contrast and boundary definition in this case. In spite of these improvements, the suppression of the echo signal in the ROI region reduces the delineation of the interior echo, resulting in possible confusion when differentiating a fibroadenoma (solid) from a cyst (liquid). This requires further investigation. Nonetheless, in this case the lesion is determined as a fibroadenoma without ambiguity based on the boundary echogenicity. Typically, a cyst has brighter echoes at the boundary, and those of a fibroadenoma are not as strong.

\section{Carcinoma}

Fig. 7 shows images of a carcinoma lesion in a 63year-old female patient. The background and the ROI used in the calculation of the $\mathrm{CR}$ and $\mathrm{CNR}$ are indicated in Fig. 7(a) by the black and white boxes, respectively. Fig. 7(a) shows the original image, and Figs. 7(b) and (c) show the corrected images obtained using the correlationbased method and the GCF-weighting method, respectively. The CRs and CNRs were $13.63 \mathrm{~dB}$ and 2.19 for the original image, $13.80 \mathrm{~dB}$ and 2.23 for the correlation-based method, and $21.52 \mathrm{~dB}$ and 2.87 for the GCF-weighting method. The CR and CNR improved by $0.16 \mathrm{~dB}$ and $1.59 \%$ with the correlation-based method and by $7.89 \mathrm{~dB}$ and $30.75 \%$ with the GCF-weighting method, respectively. Again, the original image shows an indistinct lesion margin. The internal echo and several echogenic foci indicating microcalcifications within the breast parenchyma also are not clearly evident. However, with the application of the correlation-based method, no noticeable change was observed, and the method was not able to improve the image. The GCF-weighted image shows better characterization of the margin, enhanced delineation of the internal architecture, and enhanced conspicuity of microcalcifications within the breast parenchyma due to improved spatial resolution and contrast.

\section{E. Abscess}

Fig. 8 shows images of an abscess lesion in a 31-year-old female patient. The background and the ROI used in the calculation of the CR and CNR are indicated in Fig. 8(a) by the black and white boxes, respectively. Fig. 8(a) shows the original image, and Figs. 8(b) and (c) show the corrected images obtained using the correlation-based method and the GCF-weighting method, respectively. The CRs and CNRs were $22.20 \mathrm{~dB}$ and 3.73 for the original image, $22.85 \mathrm{~dB}$ and 3.80 for the correlation-based method, and $32.11 \mathrm{~dB}$ and 4.59 for the GCF-weighting method. The CR and CNR improved by $0.65 \mathrm{~dB}$ and $1.83 \%$ with the correlation-based method and by $9.91 \mathrm{~dB}$ and $23.10 \%$ with the GCF-weighting method, respectively. The original image shows a well-circumscribed hypoechoic mass with internal echogenic debris, a slight posterior acoustic enhancement, and indistinct calcification. However, with the application of the correlation-based method, no noticeable change was observed, and the method was not able to improve the image. The GCF-weighted image shows a clearer lesion margin and enhanced delineation of the internal architecture due to improvement of the contrast.

\section{F. Milk of Calcium}

Fig. 9 shows images of a milk of calcium in a cyst lesion in a 25-year-old female patient. Fig. 9(a) shows the original image, and Figs. 9(b) and (c) show the corrected 

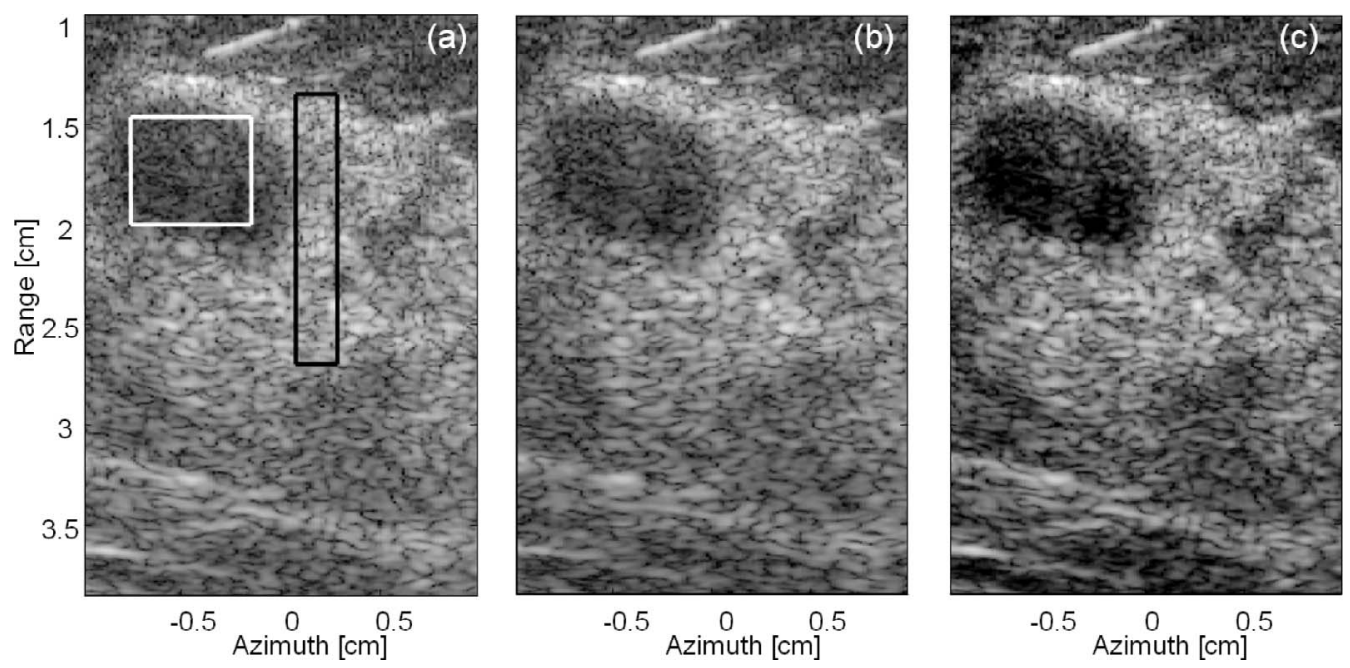

Fig. 6. Images of a fibroadenoma in a 42-year-old woman, displayed with a dynamic range of 60 dB: original B-mode image (a), and images obtained using the correlation-based method (b) and the GCF-weighting method (c). The black box in (a) indicates the background region. The white box indicates the ROI.
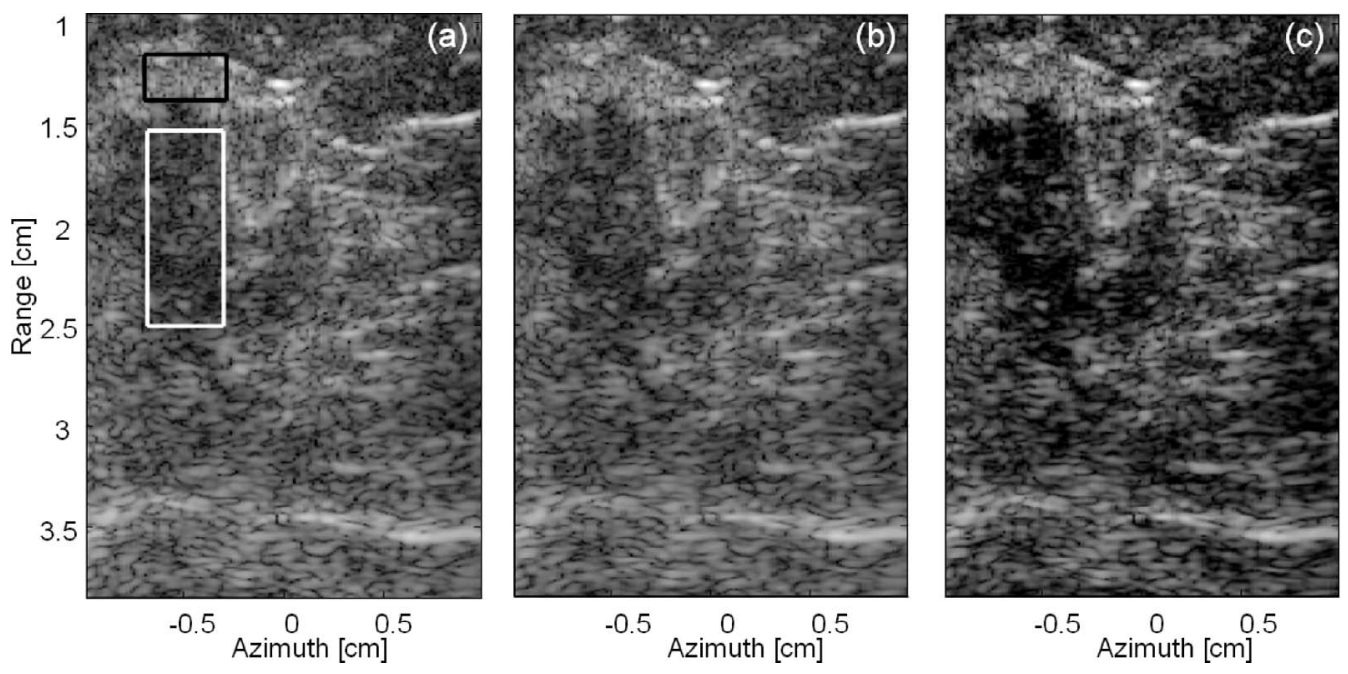

Fig. 7. Images of a carcinoma in a 63-year-old woman, displayed with a dynamic range of 60 dB: original B-mode image (a), and images obtained using the correlation-based method (b) and the GCF-weighting method (c). The black box in (a) indicates the background region. The white box indicates the ROI.
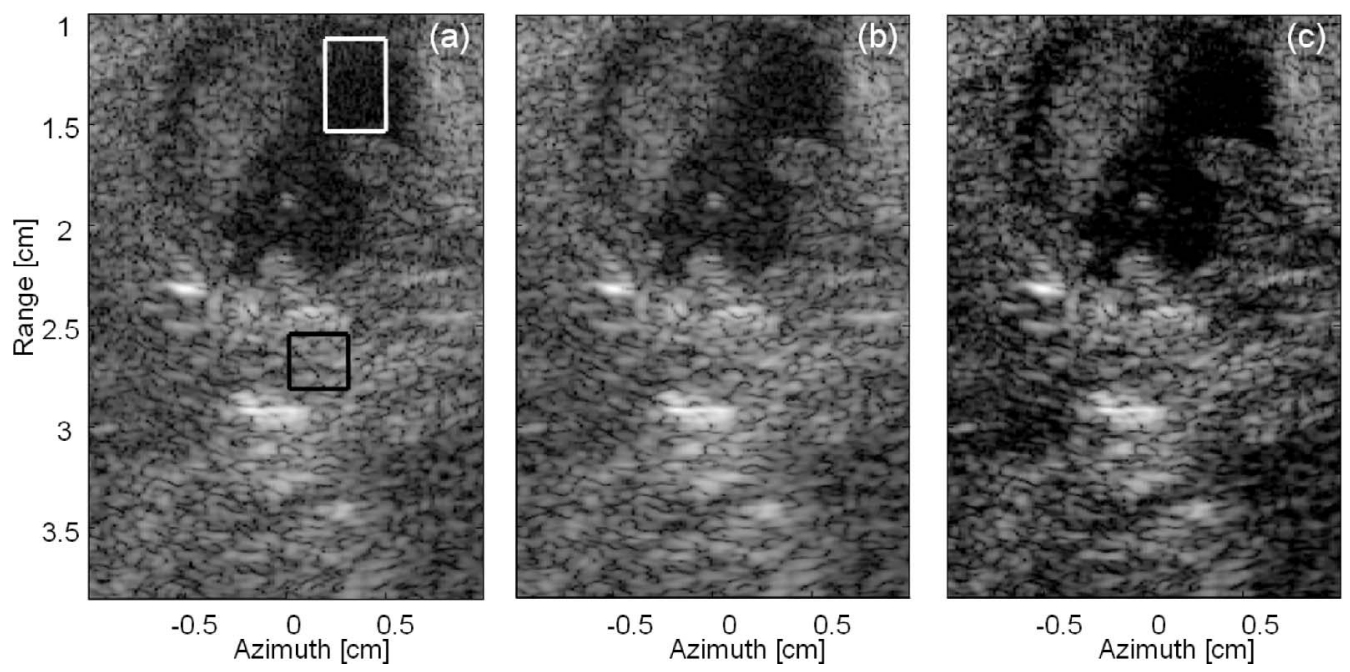

Fig. 8. Images of an abscess in a 31-year-old woman, displayed with a dynamic range of $60 \mathrm{~dB}$ : original B-mode image (a), and images obtained using the correlation-based method (b) and the GCF-weighting method (c). The black box in (a) indicates the background region. The white box indicates the ROI. 

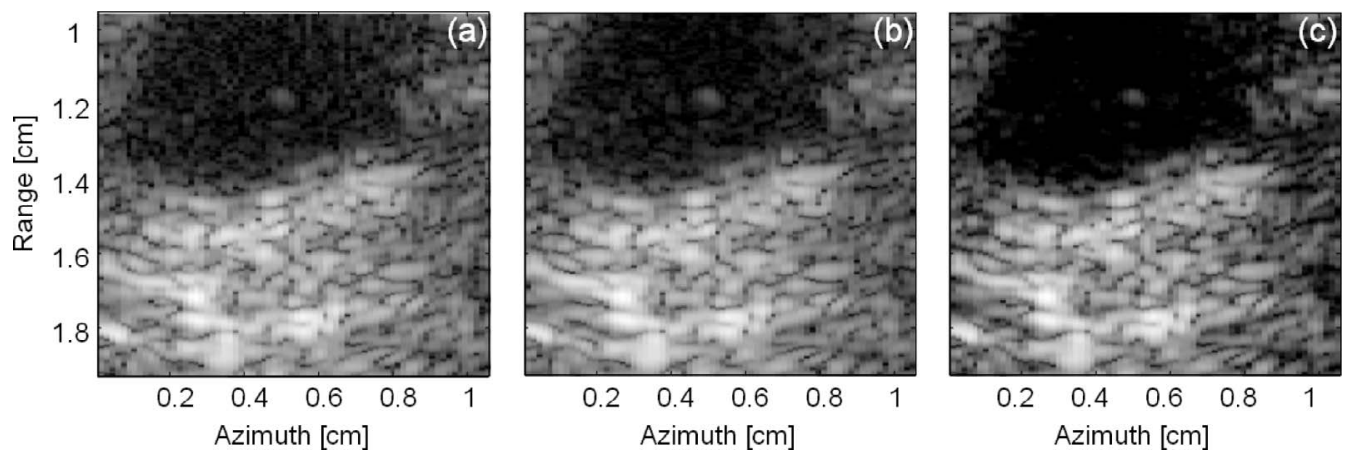

Fig. 9. Images of a milk of calcium in a cyst in a 25-year-old woman, displayed with a dynamic range of 60 dB: original B-mode image (a), and images obtained using the correlation-based method (b) and the GCF-weighting method (c).

TABLE I

Summary of the CR AND CNR VAlues.

\begin{tabular}{|c|c|c|c|c|c|c|c|c|c|c|c|c|c|}
\hline \multirow[b]{2}{*}{$\begin{array}{c}\text { Patient } \\
\text { no. }\end{array}$} & \multirow[b]{2}{*}{$\begin{array}{c}\text { Age } \\
\text { (years) }\end{array}$} & \multirow[b]{2}{*}{$\begin{array}{c}\text { Lesion } \\
\text { type }^{1}\end{array}$} & \multicolumn{3}{|c|}{$\mathrm{CR}$} & \multicolumn{3}{|c|}{ CNR } & \multicolumn{2}{|c|}{$\begin{array}{c}\text { CR } \\
\text { improvement } \\
(\mathrm{dB})\end{array}$} & \multicolumn{2}{|c|}{$\begin{array}{c}\text { CNR } \\
\text { improvement } \\
(\%)\end{array}$} & \multirow{2}{*}{$\begin{array}{c}\begin{array}{c}\text { Subjective } \\
\text { score }\end{array} \\
\text { GCF }\end{array}$} \\
\hline & & & Original & $\begin{array}{l}\text { Corr. }{ }^{I} \\
\text { based }\end{array}$ & $\mathrm{GCF}$ & Original & $\mathrm{CF}$ & $\mathrm{GCF}$ & $\begin{array}{l}\text { Corr. } \\
\text { based }\end{array}$ & $\mathrm{GCF}$ & $\begin{array}{l}\text { Corr. } \\
\text { based }\end{array}$ & $\mathrm{GCF}$ & \\
\hline 1 & 53 & Cyst & 38.84 & 38.51 & 53.19 & 5.84 & 5.82 & 6.96 & -0.33 & 14.34 & -0.28 & 19.18 & $2+$ \\
\hline 2 & 49 & Cyst & 42.00 & 41.58 & 57.05 & 6.09 & 6.37 & 7.08 & -0.41 & 15.04 & 4.51 & 16.19 & $2+$ \\
\hline 3 & 59 & $\mathrm{CA}$ & 26.00 & 26.53 & 36.70 & 4.03 & 3.99 & 4.73 & 0.52 & 10.69 & -0.88 & 17.43 & $1+$ \\
\hline 6 & 79 & $\mathrm{CA}$ & 10.39 & 11.05 & 19.57 & 1.53 & 1.59 & 2.46 & 0.65 & 9.17 & 3.85 & 60.94 & $2+$ \\
\hline 7 & 66 & Cyst & 20.97 & 20.52 & 33.53 & 3.07 & 2.95 & 4.33 & -0.44 & 12.56 & -3.85 & 40.97 & $2+$ \\
\hline 8 & 43 & $\mathrm{CA}$ & 4.35 & 4.84 & 7.67 & 0.66 & 0.73 & 0.96 & 0.49 & 3.32 & 11.65 & 45.41 & $1+$ \\
\hline 9 & 33 & Abscess & 14.11 & 14.57 & 20.05 & 2.16 & 2.23 & 2.59 & 0.46 & 5.93 & 2.96 & 19.58 & $1+$ \\
\hline 10 & 59 & $\mathrm{CA}$ & 16.37 & 17.94 & 24.87 & 2.58 & 2.79 & 3.29 & 1.56 & 8.50 & 7.92 & 27.24 & $2+$ \\
\hline 11 & 63 & FA & 15.32 & 15.28 & 20.59 & 2.67 & 2.65 & 2.99 & -0.03 & 5.27 & -0.90 & 12.05 & $1+$ \\
\hline 16 & 50 & $\mathrm{CA}$ & 21.07 & 22.07 & 30.27 & 3.55 & 3.69 & 4.21 & 0.99 & 9.19 & 3.86 & 18.36 & $1+$ \\
\hline 17 & 42 & FA & 16.36 & 16.91 & 24.12 & 2.67 & 2.73 & 3.44 & 0.54 & 7.75 & 2.23 & 28.97 & $2+$ \\
\hline 18 & 25 & Cyst & 15.45 & 19.61 & 27.09 & 2.56 & 3.12 & 3.18 & 4.16 & 11.63 & 22.09 & 24.50 & $2+$ \\
\hline 19 & 45 & $\mathrm{CA}$ & 21.56 & 21.18 & 29.26 & 3.55 & 3.29 & 3.92 & -0.37 & 7.70 & -7.26 & 10.29 & $1+$ \\
\hline 20 & 45 & $\mathrm{CA}$ & 15.85 & 16.06 & 21.33 & 2.98 & 3.07 & 3.46 & 0.21 & 5.48 & 3.17 & 16.17 & $1+$ \\
\hline 21 & 47 & Cyst & 23.10 & 23.83 & 34.91 & 3.53 & 3.74 & 4.08 & 0.73 & 11.81 & 6.02 & 15.62 & $1+$ \\
\hline 22 & 55 & FA & 24.18 & 24.33 & 33.58 & 3.80 & 3.86 & 4.31 & 0.14 & 9.39 & 1.52 & 13.30 & $1+$ \\
\hline 23 & 58 & Cyst & 24.43 & 24.20 & 35.70 & 3.45 & 3.83 & 4.32 & -0.22 & 11.26 & 11.07 & 25.18 & $1+$ \\
\hline 24 & 31 & Abscess & 22.20 & 22.85 & 30.98 & 3.73 & 3.80 & 4.37 & 0.65 & 8.78 & 1.83 & 17.12 & $1+$ \\
\hline 25 & 57 & $\mathrm{CA}$ & 26.51 & 26.92 & 34.83 & 4.14 & 4.33 & 4.56 & 0.40 & 8.31 & 4.51 & 10.02 & $2+$ \\
\hline
\end{tabular}

${ }^{1} \mathrm{CA}=$ carcinoma, $\mathrm{FA}=$ fibroadenoma, Corr. = Correlation.

images obtained using the correlation-based method and the GCF-weighting method, respectively. The contrast between the milk of calcium and the tissue improved by $0.05 \mathrm{~dB}$ for the correlation-based method and by $4.47 \mathrm{~dB}$ for the GCF-weighting method. The lateral and axial -6$\mathrm{dB}$ widths of the milk of calcium object were 0.51 and $0.39 \mathrm{~mm}$ in the original image, 0.55 and $0.41 \mathrm{~mm}$ in the correlation-based image, and 0.43 and $0.32 \mathrm{~mm}$ in the GCF-weighted image, respectively. The GCF-weighting method improved both the spatial resolution and the contrast in this case, which in turn helped in the detection of calcification.

\section{Discussion}

The CR and CNR values obtained with the correlationbased and GCF-weighting methods are listed in Table I. The mean values for the CR and CNR improvements were $0.42 \mathrm{~dB}$ and $3.4 \%$ for the correlation-based method and $8.57 \mathrm{~dB}$ and $23.20 \%$ for the GCF-weighting method. It should be noted that, although the CR and CNR are widely reported as performance indices in the literature and are directly related to fundamental characteristics of an image, their exact values may change with the selected ROI and may not be directly related to diagnostic out- 
comes. Therefore, these images also were evaluated by an experienced clinician (Y.-H. Chou) using the following scoring system: $2+$, significant improvement; $1+$, moderate improvement; 0 , no noticeable effects; $1-$, slight degradation; and 2-, significant degradation. In general, application of the GCF-weighting method improved the image quality in all the cases assessed in this study. The improvement in the contrast was more significant for a liquid lesion (e.g., a cyst) than for a solid lesion (e.g., a fibroadenoma), and the improvement in the boundary definition makes it easier to detect a malignant tumor (e.g., a carcinoma).

The proposed method requires only the use of individual channel data for each firing. Nonetheless, complete data sets were acquired in this study. With the complete data set, dynamic, two-way focusing can be realized. Therefore, the problem associated with reduced coherence at positions away from the fixed transmit focus can be avoided. However, acquisition of a complete data set suffers from lower signal-to-noise ratios and potential motion artifacts. In clinical situations, a reasonable compromise is to use multizone focusing on transmit.

Although the correlation-based method is one of the most popular adaptive imaging methods reported in the literature, several problems were encountered when we applied this to breast imaging in this study. The estimated time delays were affected by several factors, including the cumulative error along the array direction, the signal-tonoise ratio, the correlation between adjacent channels, the kernel size, and the aberrator integration errors of a 1D array [17]-[19]. In addition to the above problems, the distributed sound-velocity inhomogeneities also represent a uniquely difficult problem in adaptive breast imaging, as illustrated in Fig. 10. The image shown in Fig. 10(a) is displayed without any aberration correction. Fig. 10(b) plots the estimated time-delay errors as a function of the channel index at the image points corresponding to the boxes marked in Fig. 10(a), which are located along the same beam and are spaced by $7.7 \mathrm{~mm}$. The time delays were estimated according to previously described principles [3]. Significant variations in the estimated time delays are clearly evident, and these limit the performance of the correlation-based method. The depth dependency of the estimated time delay may result from breast tissue comprising a heterogeneous mixture of fatty, fibrotic, connective, and glandular tissues.

The arrival time and energy level reportedly vary with breast sample thickness [20], and the correlation length of the aberration profile in breast tissue varies with age and body type [20]-[22]. Therefore, it is to be expected that the near-field, phase-screen model is inaccurate, resulting in poor breast imaging when using the correlation-based method. It also has been reported that waveform distortion is severe and sidelobes are high for breast tissue, primarily because it is a highly refracting and heterogeneous medium [20], [23]-[25]. Therefore, adaptive sidelobe-reduction techniques, such as the GCF-weighting method investigated in the study, are of particular interest because they do not make any assumptions regarding the origin of the side-

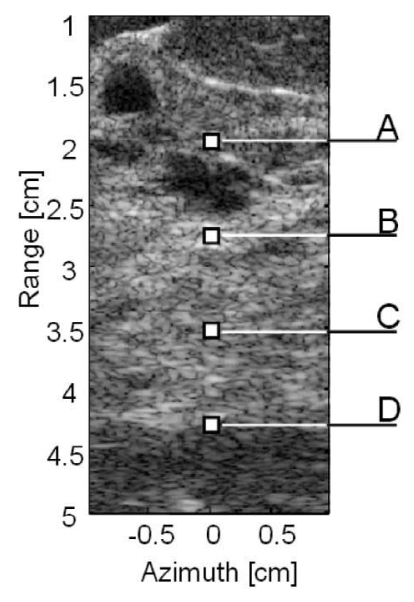

(a)

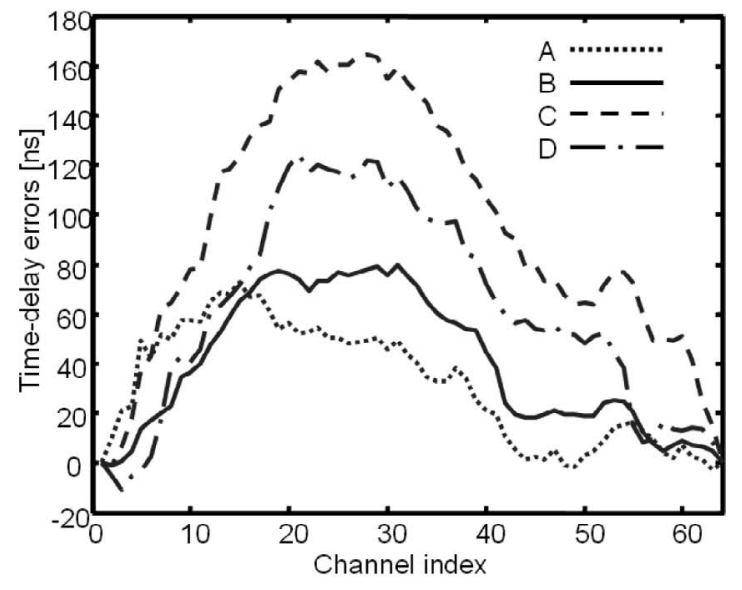

(b)

Fig. 10. Estimated time-delay errors. (a) Clinical image displayed with a dynamic range of $60 \mathrm{~dB}$. (b) Corresponding estimated timedelay errors as functions of channel index at the imaging points indicated in (a).

lobes. As shown in this paper, application of the GCFweighting method significantly improves the contrast and boundary definition for cyst and solid masses. We also have demonstrated that the GCF-weighting method is more effective than correlation-based methods in improving the quality of images obtained from ultrasonic breast imaging without the use of a 2-D (or 1.5-D) array.

This paper illustrates efficacy in sidelobe reduction of the proposed method using 1-D arrays. Nonetheless, the proposed method also can be extended to imaging with 1.5-D/2-D arrays. In particular, because the aperture also is sampled along the elevational direction, the proposed method can be applied to improve elevational focusing quality. Signal processing required for elevational GCF weighting with $1.5-\mathrm{D} / 2-\mathrm{D}$ arrays will be similar to that for lateral GCF weighting with 1-D arrays.

\section{CONCLUSIONS}

In this paper, we have experimentally evaluated an adaptive imaging approach for reducing focusing errors 
in ultrasonic imaging. This method is based on coherence and corrects focusing errors by suppressing unwanted sidelobes. The experimental results demonstrate that the image quality was noticeably improved in all of the cases investigated using a 1-D array.

\section{ACKNOWLEDGMENT}

Support from the National Science Council of Taiwan under grant no. NSC 95-2221-E-002-170 is gratefully acknowledged.

\section{REFERENCES}

[1] S. A. Goss, R. L. Johnston, and F. Dunn, "Comprehensive compilation of empirical ultrasonic properties of mammalian tissues," J. Acoust. Soc. Amer., vol. 64, pp. 423-457, 1978.

[2] S. W. Flax and M. O'Donnell, "Phase-aberration correction using signals from point reflectors and diffuse scatterers: Basic principles," IEEE Trans. Ultrason., Ferroelect., Freq. Contr., vol. 35, no. 6, pp. 758-767, 1988.

[3] M. O'Donnell and S. W. Flax, "Phase-aberration correction using signals from point reflectors and diffuse scatterers: Measurements," IEEE Trans. Ultrason., Ferroelect., Freq. Contr., vol. 35 , no. 6 , pp. $768-774,1988$.

[4] S. Krishnan, K. W. Rigby, and M. O'Donnell, "Improved estimation of phase aberration profile," IEEE Trans. Ultrason., Ferroelect., Freq. Contr., vol. 44, no. 3, pp. 701-713, 1997.

[5] G. C. Ng, S. S. Worrell, P. D. Freiburger, and G. E. Trahey, "A comparative evaluation of several algorithms for phase aberration correction," IEEE Trans. Ultrason., Ferroelect., Freq. Contr., vol. 41, no. 5, pp. 631-643, 1994.

[6] D. L. Liu and R. C. Waag, "Correction of ultrasonic wavefront distortion using backpropagation and a reference waveform method for time-shift compensation," J. Acoust. Soc. Amer., vol. 96, no. 2, pp. 649-660, 1994.

[7] P.-C. Li and M. O'Donnell, "Phase aberration correction on twodimensional conformal arrays," IEEE Trans. Ultrason., Ferroelect., Freq. Contr., vol. 42, no. 1, pp. 73-82, 1995.

[8] P.-C. Li, S. W. Flax, E. S. Ebbini, and M. O'Donnell, "Blocked element compensation in phased array imaging," IEEE Trans. Ultrason., Ferroelect., Freq. Contr., vol. 40, no. 4, pp. 283-292, 1993.

[9] P.-C. Li and M. O'Donnell, "Improved detectability with blocked element compensation," Ultrason. Imag., vol. 16, no. 1, pp. 1-18, 1994.

[10] S. Krishnan, P.-C. Li, and M. O'Donnell, "Adaptive compensation of phase and magnitude aberrations," IEEE Trans. Ultrason., Ferroelect., Freq. Contr., vol. 43, no. 1, pp. 44-55, 1996.

[11] S. Krishnan, K. W. Rigby, and M. O'Donnell, "Efficient parallel adaptive aberration correction," IEEE Trans. Ultrason., Ferroelect., Freq. Contr., vol. 45, no. 3, pp. 691-703, 1998.

[12] K. W. Hollman, K. W. Rigby, and M. O'Donnell, "Coherence factor of speckle from a multi-row probe," in Proc. IEEE Ultrason. Symp., 1999, pp. 1257-1260.

[13] R. Mallart and M. Fink, "Adaptive focusing in scattering media through sound-speed inhomogeneities: The van Cittert Zernike approach and focusing criterion," J. Acoust. Soc. Amer., vol. 96, no. 6, pp. 3721-3732, 1994.

[14] S. D. Silverstein, "Ultrasound scattering model: 2-D crosscorrelation and focusing criteria-theory, simulations, and experiments," IEEE Trans. Ultrason., Ferroelect., Freq. Contr., vol. 48, no. 4, pp. 1023-1030, 2001.

[15] P.-C. Li and M.-L. Li, "Adaptive imaging using the generalized coherence factor," IEEE Trans. Ultrason., Ferroelect., Freq. Contr., vol. 50, no. 2, pp. 128-141, 2003.

[16] W. F. Walker and G. E. Trahey, "Aberrator integration error in adaptive imaging," IEEE Trans. Ultrason., Ferroelect., Freq. Contr., vol. 44, no. 4, pp. 780-791, 1997.
[17] G. C. Ng, S. S. Worrell, P. D. Freiburger, and G. E. Trahey, "A comparative evaluation of several algorithms for phase aberration correction," IEEE Trans. Ultrason., Ferroelect., Freq. Contr., vol. 41, no. 5, pp. 631-643, 1994.

[18] W. F. Walker and G. E. Trahey, "A fundamental limit on the performance of correlation based phase correction and flow estimation techniques," IEEE Trans. Ultrason., Ferroelect., Freq. Contr., vol. 41, no. 5, pp. 644-654, 1994.

[19] F. Viola and W. F. Walker, "A comparison of the performance of time-delay estimators in medical ultrasound," IEEE Trans. Ultrason., Ferroelect., Freq. Contr., vol. 50, no. 4, pp. 392-401, 2003.

[20] L. M. Hinkelman, D.-L. Liu, R. C. Waag, Q. Zhu, and B. D. Steinberg, "Measurements and correction of ultrasonic pulse distortion produced by human breast," J. Acoust. Soc. Amer., vol. 97, no. 3, pp. 1958-1969, 1995.

[21] R. C. Gauss, G. E. Trahey, and M. S. Soo, "Adaptive imaging in the breast," in Proc. IEEE Ultrason. Symp., 1999, pp. 15631569.

[22] G. E. Trahey, P. D. Freiburger, L. F. Nock, and D. C. Sullivan, "In vivo measurements of ultrasonic beam distortion in the breast," Ultrason. Imag., vol. 13, pp. 71-90, 1991.

[23] D.-L. Liu and R. C. Waag, "Time-shift compensation of ultrasonic pulse focus degradation using least-mean-square error estimates of arrival time," J. Acoust. Soc. Amer., vol. 95, no. 1, pp. 542-555, 1994.

[24] Q. Zhu and B. D. Steinberg, "Wavefront amplitude distortion and image sidelobe levels. I. Theory and computer simulations," IEEE Trans. Ultrason., Ferroelect., Freq. Contr., vol. 40, no. 6, pp. 747-753, 1993.

[25] Q. Zhu, B. D. Steinberg, and R. L. Arenson, "Wavefront amplitude distortion and image sidelobe levels. II. In vivo experiments," IEEE Trans. Ultrason., Ferroelect., Freq. Contr., vol. 40, no. 6, pp. 754-762, 1993.

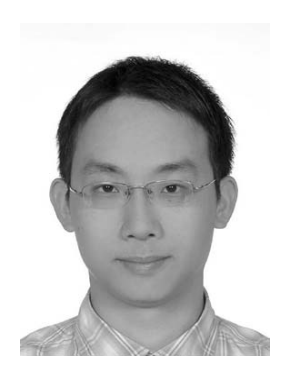

Shun-Li Wang was born in 1981 in Taipei, Taiwan, R.O.C. He received the B.S. degree in electrical engineering from National Taiwan University, Taiwan, R.O.C. in 2003. He is currently working toward the Ph.D. degree in electrical engineering at National Taiwan University. His current research interests include biomedical ultrasonic imaging, especially for flow velocity estimation and adaptive imaging.

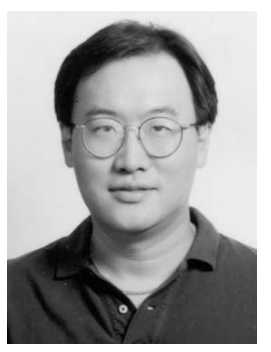

Pai-Chi Li (S'93-M'95-SM'01) received the B.S. degree in electrical engineering from National Taiwan University, Taipei, Taiwan, R.O.C., in 1987, and the M.S. and Ph.D. degrees from the University of Michigan, Ann Arbor in 1990 and 1994, respectively, both in electrical engineering: systems.

He was a research assistant with the Department of Electrical Engineering and Computer Science of the University of Michigan from 1990 to 1994. He joined Acuson Corporation, Mountain View, CA, as a member of the Technical Staff in June 1994. His work in Acuson was primarily in the areas of medical ultrasonic imaging system design for both cardiology and general imaging applications. In August 1997, he went back to the Department of Electrical Engineering at National Taiwan University, where he is currently Professor of Department of Electrical Engineering and Director of Institute of Biomedical Electronics and Bioinformatics. His current research interests include biomedical ultrasonic imaging and signal processing.

Dr. $\mathrm{Li}$ is a senior member of IEEE and Associate Editor of IEEE Transactions on Ultrasonics, Ferroelectrics, and Frequency Control. He is also on the Editorial Board of Ultrasonic Imaging. He received the 2005 Outstanding Electrical Engineering Professor Award, the 
Chinese Institute of Electrical Engineering, the 2004 Distinguished Research Award, National Science Council, the 2004 Distinguished Research Achievement Award, National Taiwan University, the 2003 Outstanding Researcher Award, National Taiwan University, the 2002 Dr. Wu Dayou Research Award from National Science Council, the 2002 Outstanding Young Electrical Engineer Award from Chinese Institute of Electrical Engineering and the Distinguished Industrial Collaboration Award of Ministry of Education. He was also the recipient of the Distinguished Achievement Award in Electrical Engineering: Systems in 1994 for his outstanding academic achievement at the University of Michigan. 